\title{
Urinary System Findings Link Group Identifier
}

National Cancer Institute

\section{Source}

National Cancer Institute. Urinary System Findings Link Group Identifier. NCI Thesaurus.

Code C162033.

A sequence of characters used to link multiple urinary system findings records to a single finding. 